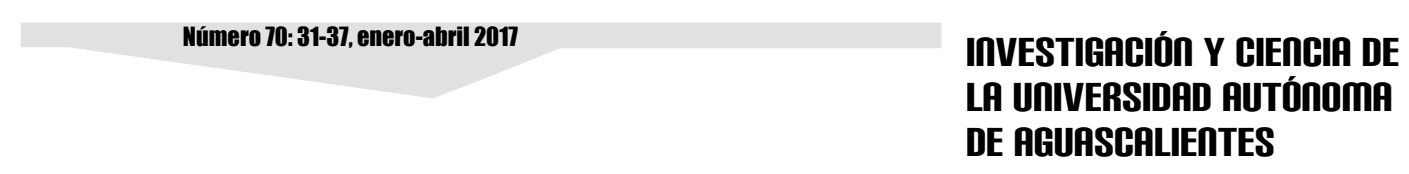

DE RGUSCHLIEITES

\title{
Influencia del magnesio y zinc en la altura de planta y verdor de hojas en Lilium
}

\author{
Influence of magnesium and zinc in plant height and leaf \\ greenness in Lilium
}

\begin{abstract}
María del Rosario Reyes Alemán ${ }^{1}$, Omar Franco Mora ${ }^{{ }^{*}}$, Edgar Jesús Morales Rosales', Delfina de Jesús Pérez López ${ }^{1}$

Reyes Alemán, M. R.., Franco Mora, O., Morales Rosales, E. J., Pérez López, D. J. Influencia del magnesio y zinc en la altura de planta y verdor de hojas de Lilium. Investigación y Ciencia de la Universidad Autónoma de Aguascalientes. Número 70: 31-37, enero-abril 2017.
\end{abstract}

RESUMEN

Se cultivó Lilium 'Acapulco' en agrolita bajo invernadero. Después de $20 \mathrm{~d}$ de plantación (DDP) y posteriormente cada 20 d hasta cosecha, se adicionaron $10 \circ 20 \mathrm{ml} / \mathrm{maceta}$ de solución Steiner aumentada 40 veces (SS $\times 40$ ); además, a partir de 35 DDP y posteriormente cada $15 \mathrm{~d}$, foliarmente se aplicaron $0-0 ; 72-80 ; 144-160 \mathrm{mg} / \mathrm{l}$ de zinc (Zn) y magnesio $(\mathrm{Mg})$, respectivamente. La SS $\times$ 40-10 $\mathrm{ml}$ generó plantas $4 \mathrm{~cm}$ más altas y con índice de verdor de hoja (IVH) similar con respecto a SS $\times$ 40-20 $\mathrm{ml}$. Zn y Mg 144 y $160 \mathrm{mg} / \mathrm{l}$ produjeron plantas $4 \mathrm{~cm}$ más altas y con 3 unidades SPAD más en relación con el control. En un segundo ciclo, a Lilium 'Serrada' fertilizada SS $\times 40-10 \mathrm{ml}$ se le aplicaron foliarmente 0-0; 72-160; 144-80; 144-160; 288-320 y 432-480 mg/l $\mathrm{Zn}$ y $\mathrm{Mg}$, respectivamente. $\mathrm{Zn}$ y $\mathrm{Mg} 72-160 \mathrm{mg} / \mathrm{l}$, respectivamente, generaron plantas $9 \mathrm{~cm}$ más altas que el control, sin diferencia para IVH.

\section{ABSTRACT}

Lilium 'Acapulco' was cropped in perlite and under greenhouse conditions. After 20 days of sowing

Palabras clave: calidad de corte, crecimiento, fotosíntesis, nutrición, sustrato.

Keywords: flower cut quality, growth, photosynthesis, nutrition, substrate.

Recibido: 3 de noviembre de 2015, aceptado: 14 de septiembre de 2016

Laboratorio de Horticultura, Centro de Investigación y Estudios Avanzados en Fitomejoramiento, Facultad de Ciencias Agrícolas, Universidad Autónoma del Estado de México, México.

* Autor para correspondencia: ofrancom@uaemex.mx
(DAS), and then every 20 days till harvest, fertilization with Steiner solution increased 40 times (SS $\times 40$ ) at 10 or $20 \mathrm{ml} /$ pot was performed; moreover, since 35 DAS, and then every 15 days, foliar application with 0-0; 72-80; 144-160 $\mathrm{mg} / \mathrm{l}$ zinc (Zn) and magnesium $(\mathrm{Mg})$, respectively was done. SS $\times 40-10 \mathrm{ml}$ generated plants $4 \mathrm{~cm}$ higher and with similar leaf green index (LGI) in relation to $S S \times 40-20 \mathrm{ml}$. Plants treated with $\mathrm{Zn}$ and $\mathrm{Mg} 144$ y $160 \mathrm{mg} / \mathrm{l}$ were $4 \mathrm{~cm}$ higher and presented 3 SPAD units more than the control. In a second crop cycle, in Lilium 'Serrada' fertilized with SS $\times$ 40-10 ml/pot, foliar was applied 0-0; 72-160; 14480; 144-160; $288-320$ and $432-480 \mathrm{mg} / \mathrm{l} \mathrm{Zn}$ and $\mathrm{Mg}$, respectively. Zn and $\mathrm{Mg}$ at $72-160 \mathrm{mg} / \mathrm{l}$ respectively, generated plants $9 \mathrm{~cm}$ higher than control; without differences in LGI.

\section{INTRODUCCIÓN}

En el Estado de México, el cultivo de lilis o lilium (Lilium spp.) se ha posicionado en los primeros cinco lugares de producción e impacto económico (SAGARPA, 2012). Sin embargo, la información sobre dosis adecuadas de fertilización aún es insuficiente, lo que propicia que en ocasiones los productores del sur del Estado de México apliquen dosis excesivas de fertilización, con lo que se genera contaminación ambiental y aumento del costo de producción (Franco Mora et al., en prensa). El correcto manejo de la nutrición vegetal, incluyendo la adición de micronutrimentos, modula aspectos de calidad ornamental; es decir, color, tamaño, olor, producción de metabolitos secundarios, vida poscosecha, etc. (Rodríguez Landero et al., 2012; Ying et al., 2014). La calidad del producto influye 
IIVESTIGACIÓn Y CIERCIA DE LA UNIVERSIDAD AUTÓNOMA en la relación costo beneficio; por ejemplo, en la Comunidad Económica Europea, la longitud del tallo de Lilium determina su clasificación en segunda (80$70 \mathrm{~cm})$, primera $(90-80 \mathrm{~cm})$, extra $(100-90 \mathrm{~cm})$ y super extra (más de $100 \mathrm{~cm}$ ) (Bañón Arias et al., 1993).

Ortega Blu et al. (2006) indicaron que las recomendaciones nutricionales varían dependiendo del cultivar de Lilium, ya que hay respuesta diferenciada por genotipo. Así, para Lilium 'Miami' y 'Navona', en función de su estado de desarrollo, se recomendó la aplicación al sustrato de nitrógeno (N), óxido de fósforo $\left(\mathrm{P}_{2} \mathrm{O}_{5}\right)$, óxido de potasio $\left(\mathrm{K}_{2} \mathrm{O}\right)$ y calcio (Ca) a $16.5,4.4,14.1$ y $2.6 \mathrm{~g} / \mathrm{m}^{2}$, respectivamente; mientras que para Lilium 'Fangio' la recomendación fue de $15.3 \mathrm{~N}, 4.3 \mathrm{P}_{2} \mathrm{O}_{5}, 16.8 \mathrm{~K}_{2} \mathrm{O}$ y $2.6 \mathrm{Ca}$ en $\mathrm{g} / \mathrm{m}^{2}$. La aplicación del fertilizante MulticoteTM (16 N: $18 \mathrm{P}_{2} \mathrm{O}_{5}$ : $21 \mathrm{~K}_{2} \mathrm{O} 16.8+3$ óxido de magnesio (MgO)) (Haifa, México) en el sustrato, al inicio del cultivo de Lilium 'Le Reve', 'Acapulco', 'Sorbonne' y 'Siberia', a dosis de 0,2 y $4 \mathrm{~g} / \mathrm{l}$, y posteriormente, durante el periodo vegetativo de la solución de Peters (15 N: $11 \mathrm{P}_{2} \mathrm{O}_{5}$ : $29 \mathrm{~K}_{2} \mathrm{O}$ ) en concentraciones de $2 \mathrm{~g} / \mathrm{l}$ incrementó el índice de verdor en las hojas, expresado en unidades del análisis del desarrollo suelo-planta, SPAD, por sus siglas en inglés (Soil Plant Analysis Development) (Treder, 2005).

Particularmente, la fertilización nitrogenada aumentó las concentraciones de terpenos, ésteres y aldehídos como 1,8-cineol, $\beta$-ocimeno, linalool, metil benzoato, 2,4 dimetil benzaldehído y nonanal en Lilium 'Starfighter' (Salazar Orozco et al., 2013). La concentración de $504 \mathrm{mM} / \mathrm{l}$ de potasio (K) en la solución nutritiva generó mayor tamaño de flor y altura del tallo en Lilium 'Arcachon' (Barrera Aguilar et al., 2013). La vida en florero se incrementó con la adición cada 20 d de $56 \mathrm{mg} \mathrm{N}$ y $68 \mathrm{mg}$ Ca por maceta en Lilium 'Menorca' (Franco Mora et al., en prensa).

La fertilización foliar en Lilium es complementaria a la adición de fertilizantes al sustrato y se recomienda, especialmente, cuando disminuyen las tasas fotosintética y de absorción de nutrimentos por las raíces (Betancourt Olvera et al., 2005). La combinación de una solución nutritiva más la aspersión de sulfato de potasio $2 \mathrm{~g} / \mathrm{l}$ y sacarosa $2 \mathrm{~g} / \mathrm{l}$ a 30 y 20 d antes de la cosecha durante el invierno, y 20 y 10 d antes en verano, mejoró el color de la flor en Lilium 'Fangio', 'Tresor', 'Brindisi' y 'Menorca' (Burchi et al., 2010). Betancourt Olvera et al. (2005), al aplicar en Lilium una fórmula foliar compuesta de $N$, fósforo $(P), K$, azufre $(S),(Z n)$, cobre $(C U)$, boro $(B)$, manganeso ( $\mathrm{Mn}$ ) y hierro (Fe), cada uno a $1 \%$, pH de 4.5 y adición de miel de abeja $0.1 \%$ como adherente, incrementó el índice de verdor de la hoja y el peso fresco de la planta; la fórmula foliar moduló los días a diferenciación floral y días a floración.

El programa de fertilización de Lilium bajo invernadero de la Universidad Autónoma del Estado de México tiene el objetivo de establecer fórmulas de fertilización que permitan incrementar la calidad hortícola de dicha especie, a la vez que comprender los efectos particulares de los nutrimentos. A las fórmulas de fertilización al sustrato implicando $\mathrm{N}$, paulatinamente se les adicionan nutrimentos vía foliar, por ejemplo, Ca y B, nutrimentos con los que se incrementó vida de florero (Franco Mora et al., 2008; Rodríguez Landero et al., 2012; Franco Mora et al., en prensa). En este reporte se analizó el efecto de la aplicación foliar de Zn y Mg, ya que existe información de los beneficios de su aplicación en cultivares de Lilium, pero es necesario ampliar el estudio en otros cultivares y zonas de producción (Betancourt Olvera et al., 2005; Barrantes Infante y Bertsch, 2012).

El Mg está involucrado directamente en la reacción fotosintética y el metabolismo glucídico en la planta, además activa enzimas que intervienen en la síntesis de los ácidos nucleicos (Devlin, 1976); por tanto, es factible suponer su injerencia en el verdor de las hojas. Particularmente en lilis, el manejo correcto del Mg puede resultar más barato que la adición de productos basados en hormonas vegetales para mantener el verdor de las hojas en poscosecha (Whitman et al., 2001). En Heliconia psittacorum la limitación del Mg deriva en falta de crecimiento, a este efecto solamente lo supera la falta de N (Matos et al., 2014). Por otra parte, la nutrición con Zn ha beneficiado el crecimiento de planta, la producción y características florales del gladiolo (Gladiolus spp.) (Saeed et al., 2013). Es un elemento activador de las enzimas deshidrogenasa, superóxido dismutasa y ARN polimerasa; además, su deficiencia reduce la fotosíntesis neta (Rashid, 1996). En cultivos no ornamentales, como trigo (Triticum aestivum L.), la aplicación foliar de $\mathrm{Zn}$ aumentó la producción $40 \%$ (Yilmaz et al., 1997). Por tanto, el objetivo de este trabajo fue determinar el efecto en tamaño de tallo y verdor de hojas de Lilium con fertilización al sustrato con diferentes dosis de la solución de Steiner (1961) y foliar a base de Mg y Zn. 


\section{MATERIALES Y METODOS}

\section{Condiciones generales del cultivo}

En la Facultad de Ciencias Agrícolas de la Universidad Autónoma del Estado de México, México, ubicada a los $19^{\circ} 24^{\prime} 29.32^{\prime \prime}$ norte y $99^{\circ} 41^{\prime} 22.39^{\prime \prime}$ oeste, se estableció Lilium 'Acapulco', calibre 18-20, del 20 de abril al 3 de agosto de 2012, y Lilium 'Serrada' calibre 14-16, del 12 de octubre al 30 de diciembre de 2013. Se emplearon bulbos vernalizados que se adquirieron en la empresa Bulbos de Holanda. El sustrato utilizado para ambos cultivares fue agrolita, se colocó en bolsas de polietileno color negro con dimensiones de $0.3 \times 0.3 \mathrm{~m}$, en cada bolsa se plantó un bulbo para cv. Acapulco y 2 bulbos para cv. Serrada. En ambos cultivares, para cada tratamiento se emplearon 50 macetas; es decir, se tuvieron 50 plantas de Lilium 'Acapulco' y 100 de Lilium 'Serrada'. Previo a la plantación, los bulbos se desinfestaron con una solución de Orthodifolathan (Captan, Pro-agro, México) $1 \mathrm{~g} / \mathrm{l}$ durante $5 \mathrm{~min}$. El diseño del invernadero (Rodríguez Landero et al., 2012) y la disposición de las macetas en ambos ciclos de cultivo permitió que el diseño experimental, en ambos ciclos, fuera completamente al azar. Para la toma de datos, de cada tratamiento, se trabajó con 10 plantas aleatoriamente.

Para evitar daños por alta intensidad luminosa, durante todo el ciclo de cultivo, las plantas se cubrieron con malla sombra $50 \%$ color negro; la intensidad luminosa promedio dentro del invernadero durante el día, a las 10:00, 13:00 y 16:00 h, respectivamente, fue de 75000, 93300 y 52000 Ix; mientras que por efecto de la malla sombra, la planta estuvo expuesta a 33000, 44600 y 22200 Ix, respectivamente. La humedad relativa varió de 35 a $65 \%$. El manejo del cultivo incluyó monitoreo y control adecuado de plagas y enfermedades y la aplicación foliar, a todos los tratamientos, de Ca $(130 \mathrm{mg} / \mathrm{l})$ y B (30 mg/l), cada $15 \mathrm{~d}$, a partir de $25 \mathrm{~d}$ después de plantación (DDP) y posteriormente cada 20 d hasta la cosecha (Rodríguez Landero et al., 2012). Se previó que la aplicación de Ca y B no coincidiera con la del Zn y Mg, nutrimentos en experimentación.

\section{Manejo del cv. Acapulco}

Una vez establecido el cultivo de Lilium 'Acapulco', el riego fue de $100 \mathrm{ml}$ por maceta, cada tercer día hasta la aparición de los primeros botones florales; posteriormente y hasta la cosecha, se aumentó a $200 \mathrm{ml}$ por maceta cada tercer día. La fertilización del sustrato se basó en la solución nutritiva de Steiner
(1961) aumentada 40 veces para igualar la dosis de $56 \mathrm{mg} \mathrm{N} / \mathrm{maceta} / 20$ días sugerida para Lilium (Franco Mora et al., 2008); aplicando $10 \mathrm{ml} /$ maceta, mientras que con $20 \mathrm{ml}$ por maceta la dosis sugerida de $\mathrm{N}$ se duplicó. La fertilización al sustrato inició a los 20 DDP y después se aplicó a intervalos de 20 d durante todo el ciclo de la planta, hasta su cosecha a los 105 DDP. Un segundo factor de estudio lo constituyó la aplicación de tres dosis de fertilización foliar a base de Zn y Mg, 0-0, 72-80 y 144-160 mg/l, respectivamente; esto a partir de los 35 DDP, cuando las hojas se encontraban ya expandidas, y posteriormente cada $15 \mathrm{~d}$ durante todo el ciclo del cultivo. La aplicación foliar de Zn y Mg se realizó con un aspersor manual mediante una barrera plástica entre tratamientos para evitar una aplicación de dosis diferente; la solución se aplicó hasta punto de goteo.

A los 35, 55, 75 y 95 DDP se realizaron cuantificaciones en la altura $(\mathrm{cm})$ de planta, desde la base del tallo (inicio del sistema radicular adventicio caulinar) hasta el ápice de la planta (incluyendo el botón floral, cuando se presentó). Por otro lado, el índice de verdor de las hojas se determinó con un medidor SPAD (Mod. 502, Konica Minolta, Japón) en hojas completamente extendidas a los $43,63,83$ y 103 DDP. Los valores se obtuvieron en cinco hojas de la parte intermedia de cada uno de los 10 tallos evaluados.

\section{Manejo del cv. Serrada}

Los riegos por maceta fueron de $100 \mathrm{ml}$ cada tercer día, desde la plantación hasta la cosecha, 75 DDP. De acuerdo con los resultados con Lilium cv. Acapulco, para Lilium cv. Serrada se experimentó solo con la fertilización foliar, pero en este caso se ampliaron los rangos de las dosis, de 0-0, 72-160, $144-80,144-160,288-320$ y $432-480 \mathrm{mg} / \mathrm{l}$ de $\mathrm{Zn}$ y $\mathrm{Mg}$, respectivamente. El diseño experimental fue completamente al azar con el mismo número de repeticiones. En 10 plantas elegidas aleatoriamente se determinó el crecimiento de planta y el índice de verdor de las hojas a los 35, 55 y 75 DDP; la medición de estos parámetros y la aplicación de nutrimentos foliares se realizó igual que en el cultivo de Lilium 'Acapulco'.

\section{Análisis estadístico}

Los datos de ambos experimentos se analizaron bajo un diseño completo al azar con el paquete estadístico SPSS. El experimento con Lilium 'Acapulco' se analizó de manera bifactorial; el factor dosis de solución Steiner (1961) con dos niveles (10 y $20 \mathrm{ml}$ por 
IIVESTIGACIÓn Y CIERCIA DE LA UחIVERSIDAD AUTÓNOTH DE RGUASCALIERTES maceta) y el factor dosis foliar de $\mathrm{Zn}$ y $\mathrm{Mg}$ con tres niveles (0-0, 72-80 y 144-160 mg/l, respectivamente). Posteriormente, se analizó de manera independiente a cada factor de estudio. Debido a que el factor dosis de solución Steiner (1961) afectó negativamente la altura de planta en Lilium 'Acapulco', el experimento de Lilium 'Serrada' solamente estudió el factor dosis de $\mathrm{Zn}$ y $\mathrm{Mg}$ foliar, con seis niveles (00, 72-160, 144-80, 144-160, 288-320 y 432-480 mg/l, respectivamente). Cuando el valor de $F$ fue significativo, la comparación de medias se realizó con la prueba de Tukey o bien, con la prueba t de Student cuando se presentaron los dos tratamientos por dosis de solución de Steiner (1961), ambas pruebas a $P$ $\leq 0.05$.

\section{RESULTADOS}

\section{Cv. Acapulco}

Tanto la dosis de Steiner (1961), como la aplicación foliar de $\mathrm{Zn}$ y $\mathrm{Mg}$, afectaron la altura de las plantas de Lilium a partir de los 55 DDP; la dosis de $20 \mathrm{ml}$ por planta de solución Steiner (1961) aumentada 40 veces, generó menor altura de planta (Tabla 1). En la misma fecha, con la aplicación de $144 \mathrm{mg} / \mathrm{Zn}$ y $160 \mathrm{mg} / \mathrm{Mg}$, las plantas tuvieron al menos $4 \mathrm{~cm}$ de mayor altura que las fertilizadas foliarmente con alguna de las otras dos dosis (Tabla 2). La interacción de los factores (1) solución Steiner (1961) por (2) aplicación foliar de $\mathrm{Zn}$ y Mg no fue significativa para la altura de planta en ninguna fecha.

Para el verdor de las hojas, el ANOVA bifactorial no mostró diferencias significativas por efecto de la dosis de Steiner (1961). Sin embargo, para la aplicación foliar de Zn-Mg, a 63 y 103 DDP, el control generó menor índice de verdor $(P \leq 0.05)$ que el observado en las hojas de Lilium 'Acapulco' fertilizado foliarmente con $144 \mathrm{mg} / \mathrm{l}$ de $\mathrm{Zn}$ y $160 \mathrm{mg} / \mathrm{l}$ de $\mathrm{Mg}$; mientras que a 103 DDP las hojas de tallos fertilizados foliarmente con $72 \mathrm{mg} / \mathrm{l}$ de $\mathrm{Zn}$ y $80 \mathrm{mg} / \mathrm{l}$ de $\mathrm{Mg}$ superaron también al control y presentaron índice de verdor en hojas, similar
Tabla 1. Altura de la planta (en cm) en Lilium 'Acapulco' en función de la dosis de fertilización con solución Steiner, aumentada 40 veces, al sustrato, cada 20 d

\begin{tabular}{|c|c|c|c|}
\hline \multicolumn{4}{|c|}{ Solución Steiner } \\
\hline $\begin{array}{c}\text { Días después } \\
\text { de plantación }\end{array}$ & $\begin{array}{c}\mathbf{1 0} \mathbf{~} \mathbf{~ l} / \\
\text { maceta }\end{array}$ & $\begin{array}{c}\mathbf{2 0} \mathbf{~} \mathbf{~ l /} \\
\text { maceta }\end{array}$ & Significancia \\
\hline 35 & 22.8 & 21.6 & NS \\
55 & 53.8 & 47.5 & $* *$ \\
75 & 89.0 & 82.3 & $* *$ \\
95 & 98.3 & 95 & $* *$ \\
\hline
\end{tabular}

Los valores son la media de 30 repeticiones, una planta por repetición. A nivel de fila, ${ }^{* *}$ significativo con la prueba $t$ de Student para $P \leq 0.01$; NS, no significativo. Elaboración propia.

al de los tallos tratados con $144 \mathrm{mg} / \mathrm{l}$ de $\mathrm{Zn}$ y $160 \mathrm{mg} / \mathrm{l}$ de Mg (Tabla 3).

\section{Cv. Serrada}

Para cv. Serrada, la altura de las plantas tratadas con 144-160 y 72-160 mg/l de Zn y Mg, respectivamente, superó al control (Figura 1). Por otro lado, de forma general, en Lilium 'Serrada' la aplicación foliar de Zn y Mg no generó efecto positivo en el índice de verdor de las hojas.

\section{DISCUSIÓN}

La solución Steiner contiene diversos nutrimentos (Steiner, 1961) y en este trabajo, al aplicar $10 \mathrm{ml}$ por maceta de dicha solución aumentada 40 veces cada $20 \mathrm{~d}$, se confirmó que la dosis de $53 \mathrm{mg} \mathrm{N} /$ maceta/20 d en Lilium (Rodríguez Landero et al., 2012) es adecuada para tener producción de calidad en cuanto a altura e índice de verdor de las hojas, al cultivar en un sustrato relativamente inerte como la agrolita. Así, con la aplicación de $20 \mathrm{ml}$ de la solución de Steiner (1961) por maceta, se duplicó la recomendación de $\mathrm{N}$ (Rodríguez Landero et al., 2012), lo cual no generó beneficio al cultivo e incluso redujo la altura de la planta. Fisiológicamente, Artacho Vargas y Pinochet Tejos (2008) indicaron que el efecto de la aplicación de $\mathrm{N}$ en tulipán (Tulipa gesneriana) en relación con la acumulación

Tabla 2. Altura de planta (en cm) en Lilium 'Acapulco' en función de la dosis foliar de Zn y Mg, aplicada a partir de 35 d de plantación y posteriormente cada $15 \mathrm{~d}$

\begin{tabular}{|c|c|c|c|c|}
\hline \multirow{2}{*}{ Zn-Mg } & \multicolumn{4}{|c|}{ Días después de plantación } \\
\cline { 2 - 5 }$(\mathbf{m g} / \mathbf{l})$ & $\mathbf{3 5}$ & $\mathbf{5 5}$ & $\mathbf{7 5}$ & $\mathbf{9 5}$ \\
\hline $0-0$ & $21.2^{\mathrm{a}}$ & $48.1^{\mathrm{b}}$ & $82.3^{\mathrm{b}}$ & $95.6^{\mathrm{b}}$ \\
$72-80$ & $21.5^{\mathrm{a}}$ & $47.1^{\mathrm{b}}$ & $83.1^{\mathrm{b}}$ & $94.1^{\mathrm{b}}$ \\
$144-160$ & $23.9^{\mathrm{a}}$ & $56.5^{\mathrm{a}}$ & $90.9^{\mathrm{a}}$ & $99.5^{\mathrm{a}}$ \\
\hline
\end{tabular}

Los valores son la media de 20 repeticiones, una planta por repetición. Medias con letras distintas en una misma columna difieren estadísticamente según la prueba de Tukey para $P \leq 0.05$.Elaboración propia. 


\section{IIVESTIGAGIÓn Y CUERCIA DE LA UחIVERSIDAD AUTÓnOMA

Tabla 3. Efecto de la fertilización foliar con zinc y magnesio en el índice de verdor de las hojas en Lilium 'Acapulco'

\begin{tabular}{|c|c|c|}
\hline $\begin{array}{c}\text { Día después de } \\
\text { plantación }\end{array}$ & $\begin{array}{c}\text { Tratamiento } \\
\text { Zn-Mg } \\
\text { (mg/l) }\end{array}$ & $\begin{array}{c}\text { Índice de verdor } \\
\text { (unidades SPAD) }\end{array}$ \\
\hline & $0-0$ & $24.1^{\mathrm{ab}}$ \\
& $72-80$ & $23.3^{\mathrm{b}}$ \\
& $144-160$ & $27.5^{\mathrm{a}}$ \\
& $0-0$ & $46.1^{\mathrm{b}}$ \\
63 & $72-80$ & $47.8^{\mathrm{ab}}$ \\
& $144-160$ & $49.9^{\mathrm{a}}$ \\
& $0-0$ & $44.6^{\mathrm{b}}$ \\
& $72-80$ & $48.2^{\mathrm{a}}$ \\
& $144-160$ & $47.0^{\mathrm{ab}}$ \\
& $0-0$ & $40.5^{\mathrm{b}}$ \\
& $72-80$ & $46.3^{\mathrm{a}}$ \\
& $144-160$ & $44.4^{\mathrm{a}}$ \\
\hline
\end{tabular}

Los valores son la media de 20 repeticiones, una planta por repetición. Medias con letras distintas en una misma columna difieren estadísticamente según la prueba de Tukey para $P \leq 0.05$. Elaboración propia.

de materia, se puede explicar con una ecuación de tercer grado. Esto quiere decir que al llegar al punto máximo, el efecto del fertilizante deja de ser positivo para pasar a negativo o, en este caso, generar menor altura. Como aspecto colateral y no involucrado en el presente estudio, se infiere que aumentar las dosis óptimas recomendadas de fertilización con $\mathrm{N}$ incrementa el costo del cultivo y el impacto ambiental, sin reflejarse positivamente en la ganancia por la venta del producto.

Por otro lado, la aplicación foliar de Zn y Mg influyó positivamente en la altura $y$, por tanto, en la calidad de los tallos de Lilium (Bañón Arias et al., 1993). La dosis de 144-160 mg/l, Zn-Mg, respectivamente, aumentó altura del tallo para ambos cultivares, en relación con el control. Es importante señalar que en Lilium 'Serrada' la aplicación de 70-160 mg/l ZnMg también incrementó la altura del tallo, y dicha altura fue similar a la observada con la dosis 144-160 mg/l, Zn-Mg, respectivamente; por tanto, para este efecto, en Lilium 'Serrada' la recomendación de Zn y Mg, basada en el costo del fertilizante debe ser, respectivamente, $70-160 \mathrm{mg} / \mathrm{l}$.

La adición de Zn incrementó la altura de planta en frijol mungo (Vigna radiata), y se explicó indicando que la traslocación del Zn dentro de

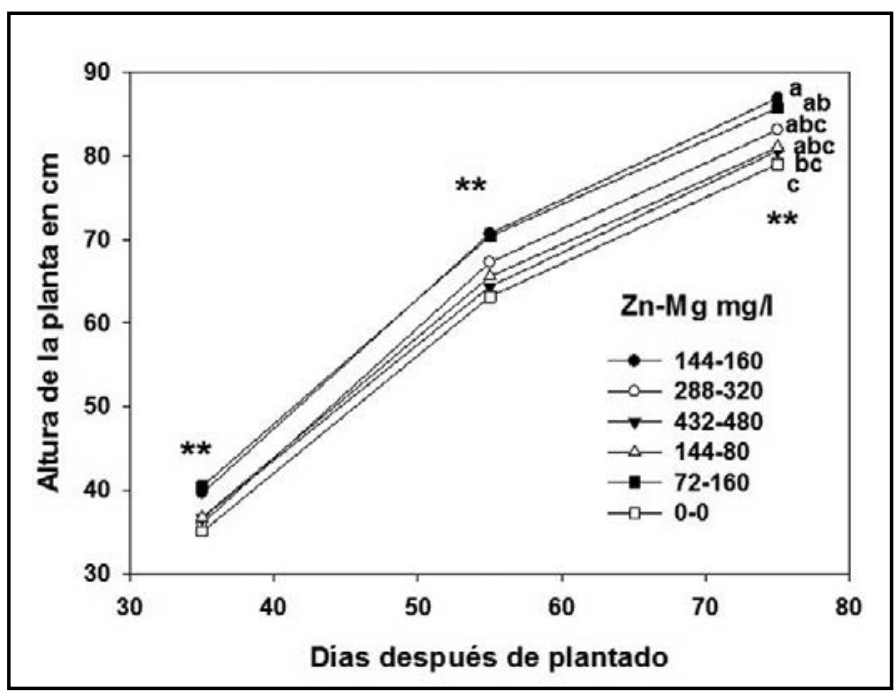

Figura 1. Cinética en el crecimiento de Lilium 'Serrada' por efecto de la aplicación foliar de Zn y Mg. Los datos son la media de 10 repeticiones \pm E. E. M. Elaboración propia.

la planta permite mayor crecimiento vegetal (Samreen et al., 2013). Particularmente, se ha indicado el efecto positivo de $\mathrm{Zn}$ en los procesos de división y, sobre todo, alargamiento celular (Clark y Zeto, 2000). Además, el Zn está relacionado con la actividad fotosintética y se sabe que la cantidad de fotosintatos está relacionada positivamente con el crecimiento vegetal (Sharma et al., 1990; Kaya et al., 2005; Nadergoli et al., 2011). En el caso del Mg se ha encontrado que una baja concentración de este elemento en cítricos (Citrus spp.) disminuye la altura de la planta (Xiao et al., 2014), además, en vid (Vitis vinifera), la limitación de Mg se relaciona con baja tasa fotosintética y producción de glucosa (Hermans y Verbruggen, 2005). De manera muy correlacionada, en el cultivo de palmarosa (Cymbopogon martinii. Roxb), la aplicación foliar de $\mathrm{Mg}$ y $\mathrm{Zn}$, con otros nutrimentos ( $\mathrm{B}$, manganeso (Mn) y hierro (Fe)), incrementó la altura de la planta y el número de hojas (Rajeswara Rao y Rajput, 2011). Esto indicaría que la correcta aplicación de ambos nutrimentos se relaciona con mayor tasa de crecimiento vegetal.

El mayor índice de verdor de las hojas de Lilium 'Acapulco' a los 63 y 103 DDP, al aplicar conjuntamente 144 y 160 mg/l Zn y Mg, respectivamente, en relación con el control, pudiera explicarse porque el Zn tiene función estructural y de componente catalítico de diversas proteínas, enzimas y coenzimas relacionadas con la síntesis de clorofila (Samreen et al., 2013); además de que Mg 


\section{INVESTIGAGIÓก Y CIECEIA DE LA UחIVERSIDAD AUTÓกOMA DE RGUASCALIERTES}

es componente central de dicho pigmento (Devlin, 1976). Para Lilium 'Acapulco' el índice de verdor de las hojas que implica mayor tasa fotosintética ayuda a explicar el mayor desarrollo vegetal, en este caso, del tamaño del tallo (Whitman et al., 2001; Rajeswara Rao y Rajput, 2011) por aplicación de Zn y Mg. Sin embargo, en Lilium 'Serrada' esto no ocurrió, ya que el índice de verdor de las hojas no fue modificada por la aplicación de los nutrimentos en estudio. Ortega Blu et al. (2006) encontraron que los efectos positivos de una fertilización para un cultivar podrían no presentarse en otro. O bien, que para Lilium 'Serrada' la adición de Zn puede alterar el equilibrio con otros nutrimentos, particularmente Fe y, por tanto, afectar la síntesis de clorofila (Rosen et al., 1977). El no observar efectos positivos de Mg y Zn en el índice de verdor de las hojas del segundo cultivar, pero sí en el largo del tallo, sugiere que el tamaño tiene influencia también de otros factores, posiblemente hormonales y/o ambientales (Whitman et al., 2001).

\section{CONCLUSIONES}

La aplicación de $10 \mathrm{ml} / \mathrm{maceta} / 20$ días de solución Steiner (1961) aumentada 40 veces para generar una dosis de $\mathrm{N}$ de $53 \mathrm{mg} \mathrm{N} / \mathrm{maceta} / 20$ días generó mayor altura de tallo en Lilium 'Acapulco', en comparación con una dosis de $20 \mathrm{ml} /$ maceta/10 días. No existió diferencia en el índice de verdor de

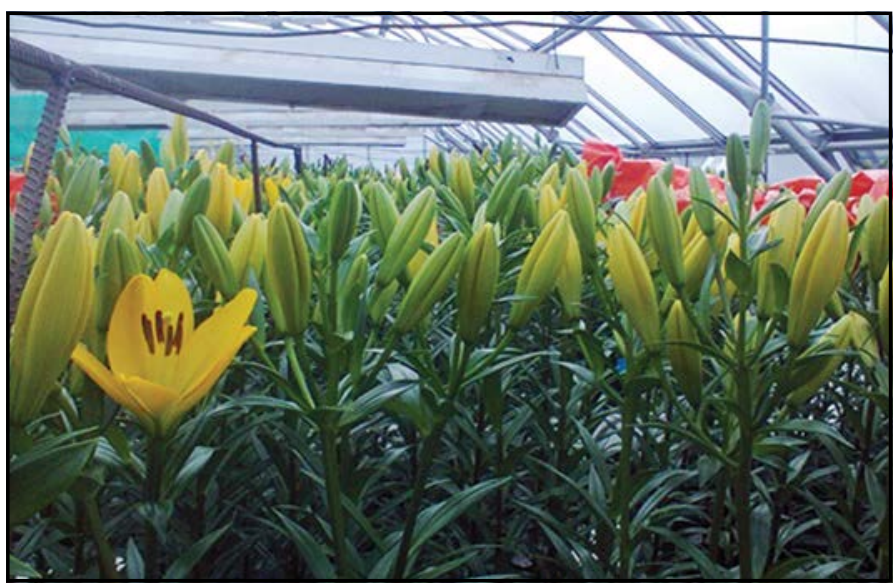

Figura 2. Se estudia la influencia del Mg y el Zn en características de Lilium. Fotografía proporcionada por María del Rosario Reyes Alemán.

las hojas por la aplicación de dichas dosis. Por otro lado, la aplicación foliar conjunta de $144 \mathrm{mg} / \mathrm{l}$ de $\mathrm{Zn}$ y de 160 mg/l de Mg en Lilium 'Acapulco' y 'Serrada', cada 15 d a partir de 35 DDP y hasta la cosecha, aumentó el tamaño del tallo en relación con el control. En el segundo cultivar, la dosis foliar de $72 \mathrm{mg} / \mathrm{l}$ de $\mathrm{Zn}$ y $160 \mathrm{mg} / \mathrm{l}$ de $\mathrm{Mg}$ también generaron mayor altura de tallo, y con el fin de no aumentar costo de cultivo se recomienda su aplicación comercial. En Lilium 'Acapulco', la aplicación foliar de Zn y Mg incrementó el índice de verdor de las hojas, pero en Lilium 'Serrada' no presentó este efecto.

LITERATURA CITADA

- $\quad$ ARTAChO VARGAS, P. y PINOChet TEJOS, D. Producción de materia seca y absorción de nitrógeno del cultivo del tulipán (Tulipa gesneriana L). Agrociencia, 42(1): 37-45, 2008.

- BAÑÓN ARIAS, S. et al. Gerbera, Lilium, tulipán y rosa. España: Mundi-Prensa, 250 pp., 1993.

- BARRANTES INFANTE, B. L. y BERTSCH, F. Curvas de absorción de nutrimentos para tres variedades de lirios (Lilium sp.) y afinamiento del programa de fertilización en una finca comercial en Heredia, Costa Rica. Agronomía Costarricense, $36(2):$ 47-60, 2012.

- BARRERA AGUILAR, E. et al. Potassium nutrition in Lilium: Critical concentrations, photosynthesis, water potential, leaf anatomy, and nutrient status. HortScience, 48(12): 1537-1542, 2013.

- Betancourt OlVERA, M. et al. Fertilización foliar una herramienta en el desarrollo del cultivo de Lilium cv. Stargazer.
Revista Chapingo Serie Horticultura, 11 (2): 371-378, 2005.

- $\quad$ BURCHI, G. et al. Improvement of flower color by means of leaf treatments in Lily. Scientia Horticulturae, 125(3): 456-460, 2010.

- CLARK, R. B. y ZETO, S. K. Mineral acquisition by arbuscular mycorrhizal plants. Journal of Plant Nutrition, 23(7): 867-902, 2000.

- DEVLIN, R. M. Fisiología vegetal (pp. 193-194). España: Omega, 1976.

- FRANCO MORA, O. et al. Incremento en la vida en florero de Lilium 'Menorca' por nitrato y óxido de calcio. En R. Rueda Luna (Ed.), Estrategias de producción en sistemas agropecuarios. México: BUAP (en prensa).

- $\quad$ FRANCO MORA, O. et al. Vida en florero de Lilium x 'Brindisi' y 'Menorca' fertilizado con nitrato y óxido de calcio. Ciencias Agrícolas Informa, 18, 4-12, 2008. 


\section{IIVESTIGAGIÓn Y CUERGIA DE LA UחIVERSIDAD AUTÓnOMH DE RGUASCHLIETTES}

- HERMANS, C.y VERBRUGGEN, N. Physiological characterization of $\mathrm{Mg}$ deficiency in Arabidopsis thaliana. Journal of Experimental Botany, 56 (418): 2153-2161, 2005.

- KAYA, M. et al. Effect of pre-sowing seed treatment with zinc and foliar spray of humic acids on yield of common bean (Phaseolus vulgaris L.). International Journal of Agriculture \& Biology, 7, 875-878, 2005.

- MATOS, V. I. J. et al. Growth and visual symptoms of macronutrient deficiency and zinc in Heliconia psittacorum cv. Golden torch. Journal of Food, Agriculture and Environment, 12(1): 169-173, 2014.

- NADERGOLI, M. S. et al. Effect of zinc and manganese and their application method on yield and yield components of common bean (Phaseolus vulgaris L.) cv. Khomein. MiddleEast Journal of Science Research, 8(5): 859-865, 2011.

- ORTEGA BLU, R. et al. Determinación de las curvas de acumulación de nutrientes en tres cultivares de Lilium spp. para flor de corte. Agrociencia, 40(1): 77-88, 2006.

- $\quad$ RAJESWARA RAO, B. R. y RAJPUT, D. K. Response of palmarosa \{Cymbopogon martinii (Roxb.) Wats. var. motia Burk.\} to foliar application of magnesium and micronutrients. Industrial Crops \& Products, 33(2): 277-281, 2011

- RASHID, A. Secondary and micronutrients. En E. S. Bantel (Ed.), Soil Science (pp. 341-386). Islamabad, Pakistan: National Book Foundation, 2005.

- RODRíGUEZ LANDERO, A. C. et al. Efecto del 1-MCP en la vida poscosecha de Lilium spp. fertilizado foliarmente con calcio y boro. Revista Mexicana de Ciencias Agrícolas, 3(8): 1623$1628,2012$.

- ROSEN, J. A. et al. Zinc, iron, and chlorophyll metabolism in zinc-toxic corn. Plant Physiology, 59(6): 1085-1087, 1977.

- SAEED, T. et al. Zinc augments the growth and floral attributes of gladiolus, and alleviates oxidative stress in cut flowers. Scientia Horticulturae, 164, 124-129, 2013.
- sagarpa (SECRetaría de agricultura, ganadería, DESARROLLO RURAL, PESCA y ALIMENTACIÓN). Garantizada la disponibilidad de flores para cubrir la demanda nacional. Comunicado de prensa 098/12. D. F., México, 14 de febrero de 2012.

- salazar orozco, G. et al. Influencia de la fertilización nitrogenada y potásica en la calidad aromática de flores de Lilium 'Starfighter'. Información Técnica Económica Agraria, 109(1): 3-12, 2013.

- $\quad$ SHARMA, P. N. et al. Zinc deficiency and pollen fertility in maize (Zea mays). Plant and Soil, 124 (2): 221-225, 1990.

- STEINER, A. A. A universal method for preparing nutrient solutions of a certain desired composition. Plant \& Soil, 15(2): 134-154, 1961.

- TREDER, J. Growth and quality of oriental lilies at different fertilization levels. Acta Horticulturae, 673, 297-302, 2005.

- WHITMAN, C. M. et al. GA4+7 plus benzyladenine reduce foliar chlorosis of Lilium longiflorum. Scientia Horticulturae, 89(2): 143-154, 2001.

- XIAO, J. X. et al. Effects of low magnesium and an arbuscular mycorrhizal fungus on the growth, magnesium distribution and photosynthesis of two citrus cultivars. Scientia Horticulturae, 177, 14-20, 2014.

- YILMAZ, A. et al. Effect of different zinc application methods on grain yield and zinc concentration in wheat cultivars grown on zinc-deficient calcareous soils. Journal of Plant Nutrition, 20(4-5): 461-471, 1997.

- YING, K. et al. Floral scent composition of Lilium 'Regale' Wilson. Acta Horticulturae, 1027, 81-86, 2014.

\section{De páginas electrónicas}

- SAMREEN, T. et al. Zinc effect on growth rate, chlorophyll, protein and mineral contents of hydroponically grown mungbeans plant (Vigna radiata). Arabian Journal of Chemistry, 2013. doi: 10.1016/j.arabjc.2013.07.005 